\title{
Experimental Investigation of Speckle Pattern by Laser Scribing for Digital Image Correlation
}

\author{
Chao-Ching Ho*, Dung-ShengWu**, Yuan-Jen Chang**, Jin-Chen Hsu**, Chia-Lung Kuo**, and Shih-Kang Kuo ${ }^{* * *}$ \\ * Graduate Institute of Manufacturing Technology and Department of Mechanical Engineering, Na- \\ tional Taipei University of Technology, Taipei 10608, Taiwan \\ E-mail: HoChao@mail.ntut.edu.tw \\ ** Department of Mechanical Engineering, National Yunlin University of Science and Technology, \\ Douliou, Yunlin 64002, Taiwan \\ *** Green Energy and System Integration Research and Development Department, China Steel Cor- \\ poration, Kaohsiung 81233, Taiwan
}

\begin{abstract}
In this work, we propose an experimental investigation of speckle pattern creation techniques for strain measurements in a stainless steel plate using digital image correlation (DIC). DIC is a non-contact full-field measurement of deformations at the surface of objects. To obtain reliable and accurate measurements, the object surface must contain sufficient speckle pattern. To investigate and assess the quality of speckle patterns produced by novel laser scribing for DIC, two speckle patterns creation techniques were also compared, i.e., grinding, and spray painting with an airbrush. Laser-produced speckle patterns were generated by a programmable galvanometer scanner guiding the beam creating speckle pattern at high speed over the stainless steel plate. In this work, four appropriately scribed speckle patterns created with different laser scribing parameters were investigated. Experimental results reveal that the laser-produced speckle pattern has an accuracy of $19 \mathrm{~nm}$ in static error measurements with an uncertainty of $3 \mathrm{~nm}$ and an uncertainty of $99 \mathrm{~nm}$ in $5 \mu \mathrm{m}$ displacement measurements.
\end{abstract}

DOI: 10.2961/jlmn.2017.02.0009

Keywords: digital image correlation, speckle pattern, mean intensity gradient, laser-scribing, fullfield measurement

\section{Introduction}

Digital image correlation (DIC) is an optical strainmapping technique that measures the deformation directly on the surface of interest. DIC is a widely used method for the full-field deformation measurement technique in experimental strain analysis [1]. To perform DIC, images composed of a random speckle pattern on the surface of the material are acquired, and then the local displacements at the surface of the material are calculated. The speckle pattern produced on the surface of the material, which provides information regarding the deformation, is the key element for achieving an accurate DIC result. In [2], it was shown that the physical properties of the pattern have a significant influence on the measurement precision. Therefore, the region of interest must contain sufficient features that it can correlate unambiguously with the reference and deformed images [3]. In [4], it is reported that the accuracy of the deformation measurement using DIC correlates highly to the condition of the speckle pattern. To perform DIC techniques for specimens on the millimeter scale, it is necessary to create a characteristic pattern on the specimen surface. Different methods for producing speckle patterns were used, such as deposition of toner particles [13], grinding [5], and paint deposition [6]. Laser scribing has been used in various manufacturing applications, as well as for marking identifications onto surfaces [7]. By applying a laser to produce the markers or grids on the surface of interest that is then tracked, the main advantage of this tech- nique is the laser can vary the speckle patterns according to the scale. The pattern design produced with laser scribing is parametric and hence can be scaled in size to provide different fields of view for different strain levels.

For this purpose, three types of microscale speckle patterns were fabricated on the specimen for microscale DIC: patterns produced with laser scribing, patterns produced with grinding, and patterns produced with spray painting. Speckle patterns produced with grinding and spray painting were used as comparative assessment tool to evaluate the performance of laser patterning method for DIC measurements. With these speckle patterns, the displacement experiments were conducted. The quality of the patterns produced was evaluated in terms of the mean intensity gradient and the entropy. The speckle patterns were examined with a self-programmed DIC system and the statistical distribution of speckle patterns was analyzed. The aim of this study is to produce an optimal micron-scale speckle pattern with laser scribing under an optical microscope, and to investigate the influence of different speckle patterns on the displacement measurement accuracy of DIC.

\section{Assessment of Patterning Techniques}

Speckle patterns produced using three different methods i.e., laser scribing (Figs. 1 and 2), grinding, and spray painting (Fig. 3) were used in the following experiments along with their 8-bit histograms (0-255 gray level range). For the investigation of the influence of the laser scribing 
parameters, such as laser energy and scribing angle, on the inherent errors of DIC, four different laser-scribing strategies were implemented. Patterns produced with laser scribing can be further divided into four subtypes: normal grid, $45^{\circ}$ grid, varied grid, and spot grid. The speckle patterns were fabricated using a fiber-optic-delivered 20-W continuous-wave (CW) laser with a wavelength of $1060 \mathrm{~nm}$ (SPI, redENERGY fiber laser). As shown in Fig. 1(a) and 1(b), normal grid and $45^{\circ}$ grid patterns were produced at a fixed scanning speed $(50 \mathrm{~mm} / \mathrm{s})$ and a fixed laser power $(6 \mathrm{~W})$. The distance between scribe lines (pitch) is $70 \mu \mathrm{m}$. As shown in Fig. 1(c), a varied grid pattern was produced at a different scanning speed (50-65 mm/s) and different laser power (5-6 W). The distance between scribe lines (pitch) varies from $70-85 \mu \mathrm{m}$. As illustrated in Fig. 1(d), a spot grid was produced at a fixed laser power $(6 \mathrm{~W})$ as a comparison basis.

Patterns produced with grinding: grit-400 sandpaper is used to manually grind the top surface and contrast was generated owing to the different levels of scattering. The contrast was used as a random pattern [3].

Spray painting with the airbrush: an airbrush gun is often used to produce a speckle pattern. However, the quality of the speckle pattern is operator-dependent and the spraying operation is a time-consuming task [6].

It can be seen from Figs. 1, 2, and 3 that the appearances and histogram distributions of these test speckle pattern images are clearly different. The histogram gives an indication of the distribution of the grayscale values. A larger distribution of intensity values indicates higher gradients and, therefore, better pattern quality [8]. It can be observed that patterns produced with laser scribing yield a broader distribution of the histogram.

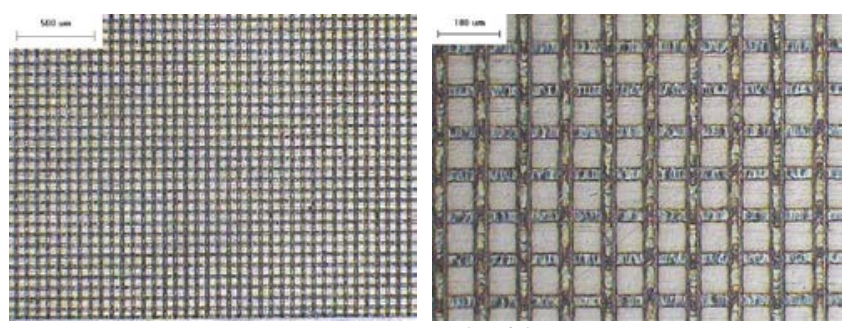

(a) Normal grid

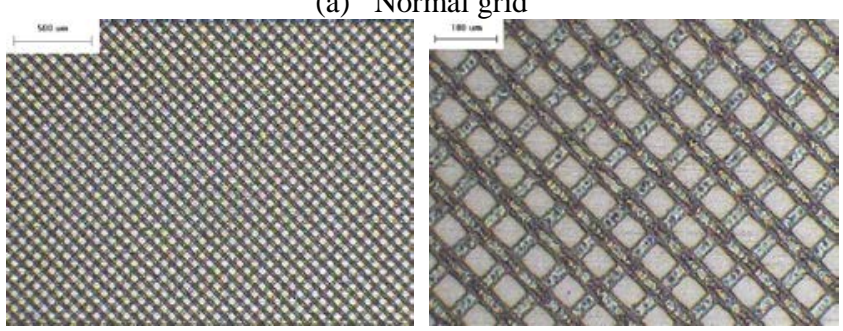

(b) $45^{\circ}$ grid

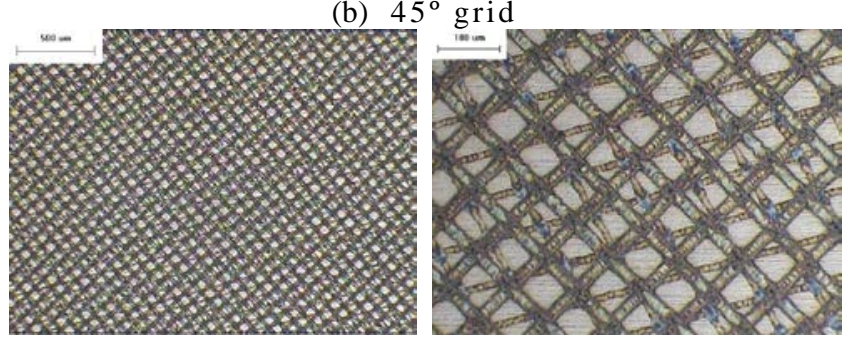

(c) Varied grid

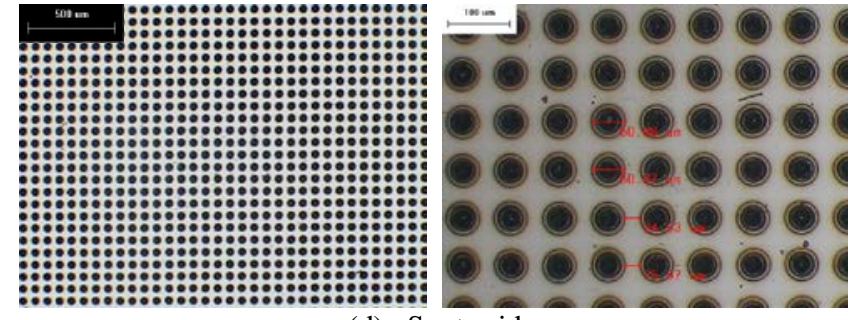

(d) Spot grid

Fig. 1 Four subtypes of speckle patterns produced with laser scribing.

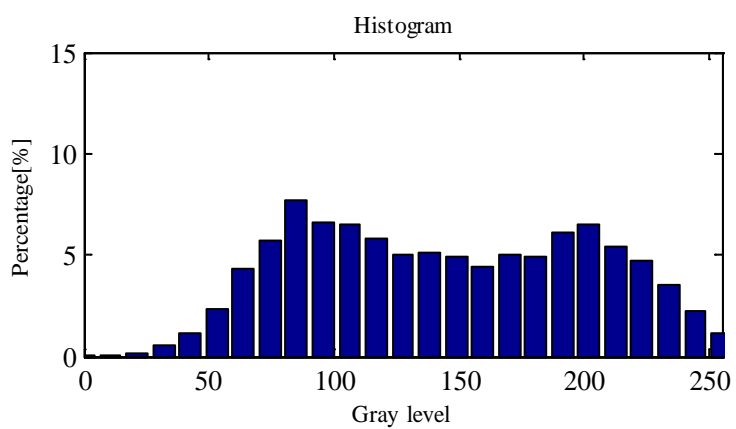

(a) Normal grid

Histogram

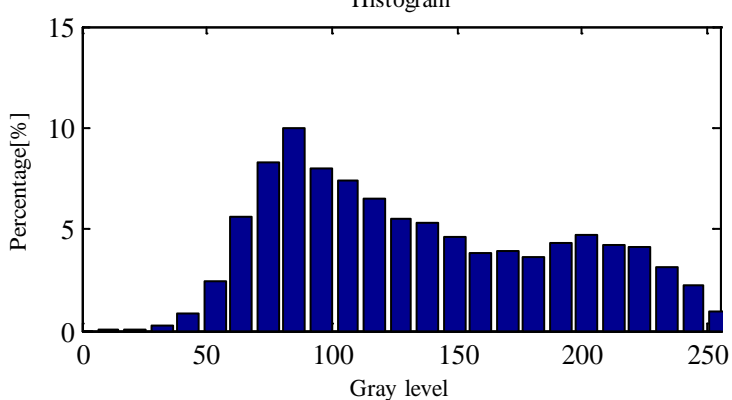

(b) $45^{\circ}$ grid

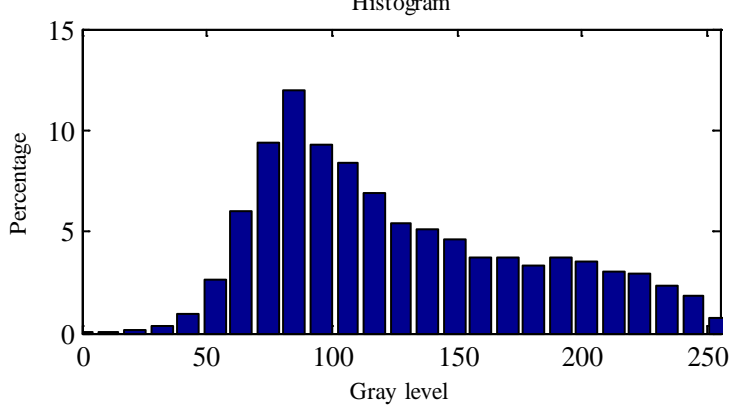

(c) Varied grid Histogram

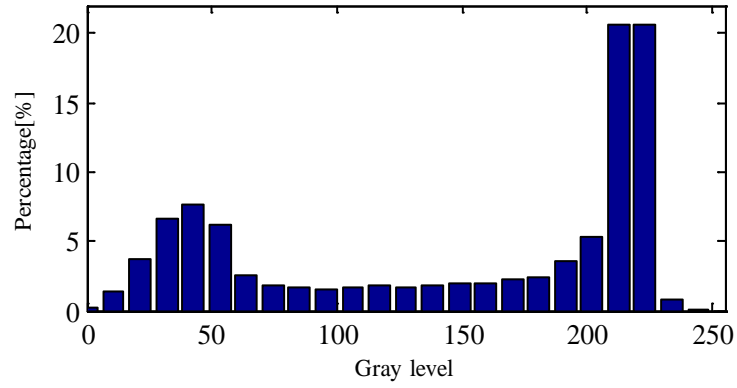

(d) Spot grid

Fig. 2 Corresponding histograms of four subtypes of speckle patterns produced with laser scribing. 


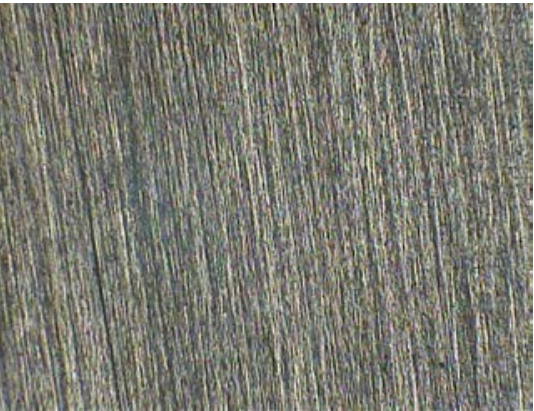

(a) Patterns of grinding

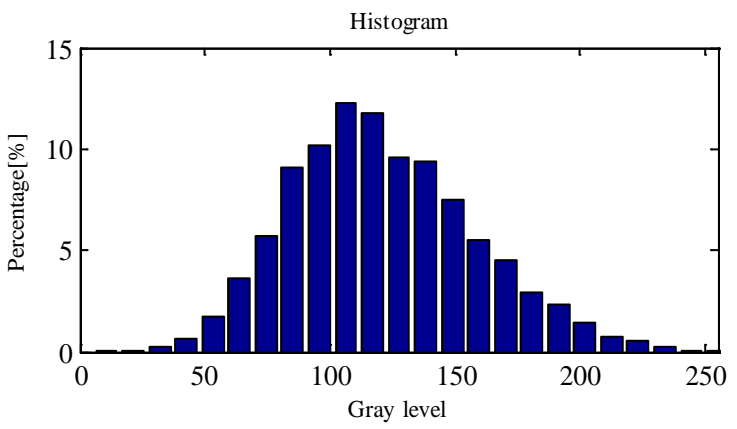

(b) Histogram of grinding

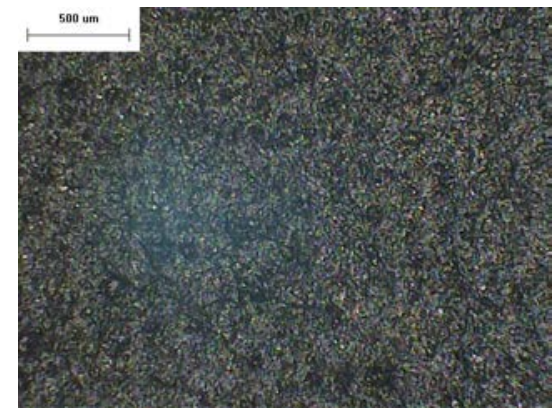

(c) Patterns of spray painting

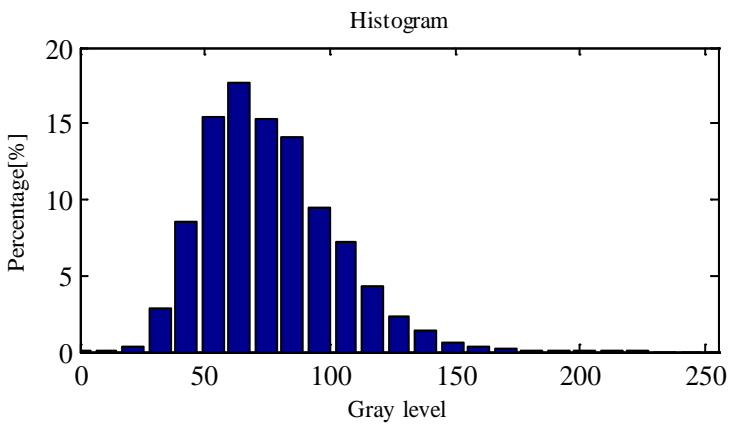

(d) Histogram of spray painting

Fig. 3 Speckle patterns produced with grinding and spray painting, and their corresponding histograms.

To reduce the uncertainty in matching the speckle pattern from the reference image to the deformed image, the speckle pattern should contain considerable contrast variation and randomness. Different patterning techniques were assessed, generating two pattern quality measurement parameters i.e., mean intensity gradient for contrast variations and entropy parameter for randomness. These parameters define the quality of different patterning techniques. A summary of the assessment of different patterning techniques is provided in Table 1.
Entropy is a statistical evaluation of texture that can be used to characterize the randomness of the input image. Entropy provides random gray level variations [9]. Hence, a speckle pattern with a high entropy value indicates a high randomness level in the gray scale distribution of the image. Speckle patterns with a high randomness performed better in terms of correlation matching [10]. The entropy assessment evaluation was applied to the speckle patterns produced using the three different methods and the four subtypes produced with laser scribing. As listed in Table 1, the entropy analysis shows that the laser-scribing line pattern i.e., normal grid, $45^{\circ}$ grid, and varied grid yield similar entropy values, which are the highest of all of the methods. The laser-scribing spot pattern and grinding pattern reach similar entropy values. The entropy value of spray painting is the lowest. The laser scribing causes nonlinear absorption of the laser radiation on the material surface; thus, this affects the results of the non-uniform heating and melting of the surface and presents an inhomogeneous surface pattern [11]. It is shown that laser scribing is beneficial in producing a highly diverse speckle pattern.

The quality of the patterns was also evaluated in terms of the mean intensity gradient (MIG, i.e., $\delta_{\mathrm{f}}$ ) [12]. The MIG of the speckle pattern was computed using the Sobel gradient operator to evaluate the quality of the entire speckle pattern in this work. The speckle pattern with a large mean intensity gradient indicates a larger variation of intensity, i.e., pattern with a higher contrast and higher density. Table 1 shows the MIG values of each speckle pattern, which was computed for the quality assessment of the speckle patterns used in DIC. The MIG of the speckle pattern produced with laser scribing is significantly larger than that of other speckle patterns, whereas the speckle pattern produced with spray painting shows the lowest mean intensity gradient. Higher MIG values indicate higher contrast and higher density of speckles. This is in good agreement with the results obtained from the entropy value.

Table 1 Assessment with different patterning techniques

\begin{tabular}{rcc}
\hline Patterning techniques & \multicolumn{2}{c}{ Assessment } \\
\cline { 2 - 3 } & MIG & Entropy \\
\hline Laser scribing & & \\
Normal grid & 15.75 & 7.7 \\
$45^{\circ}$ grid & 24.31 & 7.6 \\
Varied grid & 26.15 & 7.6 \\
Spot grid & 13.06 & 7.1 \\
\hline Grinding & 12.08 & 7.2 \\
\hline Spray painting & 11.41 & 6.6 \\
\hline
\end{tabular}

\section{Experimental Results}

The images acquired with optical microscopy can be incorporated with DIC to measure the strain distribution. Images at a magnification of $4.06 \mu \mathrm{m} /$ pixel with a size of $640 \times 480$ pixel were captured at room temperature. The area of the pattern acquired for DIC analysis was $20 \times 20$ $\mathrm{mm}^{2}$.

Initially, the static error estimation was considered to examine the inherent errors of each speckle pattern caused 
by random error and lighting variation. As shown in Fig. 4, the reference and deformed images are acquired at a static state i.e., without movement. Experimental results show that the inherent errors of the speckle pattern produced with laser scribing (varied grid) are considerably lower those that of other speckle patterns, whereas the speckle pattern produced with spray painting shows the highest static error. It is observed that the speckle pattern with the higher mean intensity gradient produces a smaller static error. The good agreement between the MIG, entropy values, and static error proves the effectiveness of the speckle pattern produced with laser scribing.

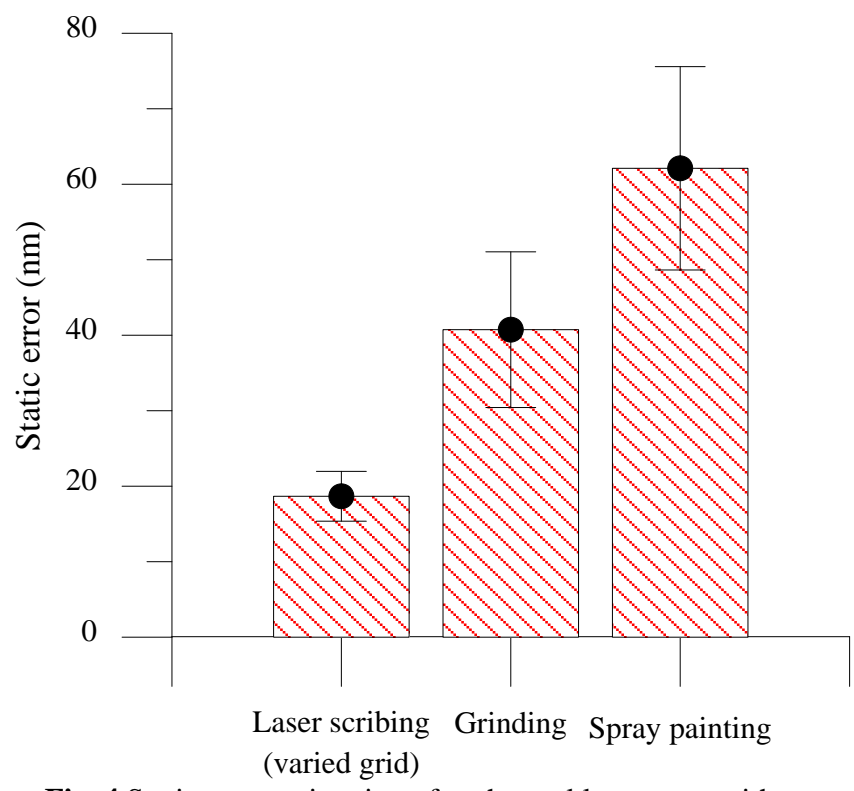

Fig. 4 Static error estimation of each speckle patterns without movement.

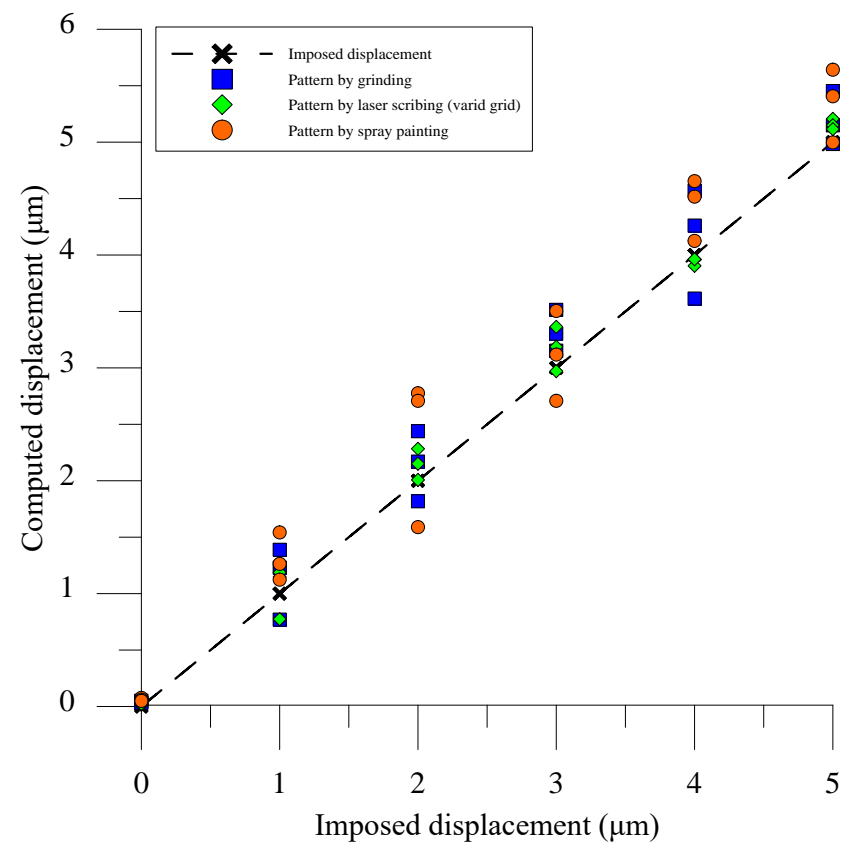

Fig. 5 Displacement field computed using DIC and real imposed displacement (an increment of $1 \mu \mathrm{m}$ ).

The accuracy of the produced speckle patterns were verified with displacement tests. The accuracy of translation in DIC was evaluated using the fabricated pattern con- sidering the disturbance generated from the actual observation. Each fabricated pattern was translated in the $X$ direction from 1 to $5 \mu \mathrm{m}$. Displacements were imposed by a micro-translation stage to perform $1 \mu \mathrm{m}$ increments. The positioning accuracy of the micro-translation stage is 0.5 $\mu \mathrm{m}$. The translation was measured by correlating the original image and the translated image using DIC. The estimated displacements using DIC in this work were compared with the exact imposed translation values. Deformed images were obtained with a plane translation in the horizontal direction ( $X$-direction) and a zero translation along the vertical direction ( $Y$-direction). The subset size used was $61 \times$ 61 pixel. Fig. 5 shows the relation between the displacement fields computed using DIC and the actual displacement in the $X$-direction. Comparisons between different measurements show absolute average errors of $104 \mathrm{~nm}$ for laser scribing, $188 \mathrm{~nm}$ for grinding, and $312 \mathrm{~nm}$ for spray painting. In terms of the displacement results, the predicted displacements by the speckle patterns produced with laser scribing were in the best agreement with the actual translation.

Fig. 6 shows the standard deviation of the measured displacement for each speckle pattern, which reflects the deviation of the measured displacement corresponding to the mean value. As illustrated, the standard deviation of the speckle pattern produced with laser scribing is considerably lower than that of other speckle patterns, whereas the speckle pattern produced with spray painting shows the highest standard deviation. It was observed that the speckle pattern with a higher mean intensity gradient produces smaller standard deviation. The good agreement between MIG, entropy values, and standard deviation proves the effectiveness of the speckle pattern produced with laser scribing. It is shown that the laser scribing has a significant beneficial effect on the overall measurement error.

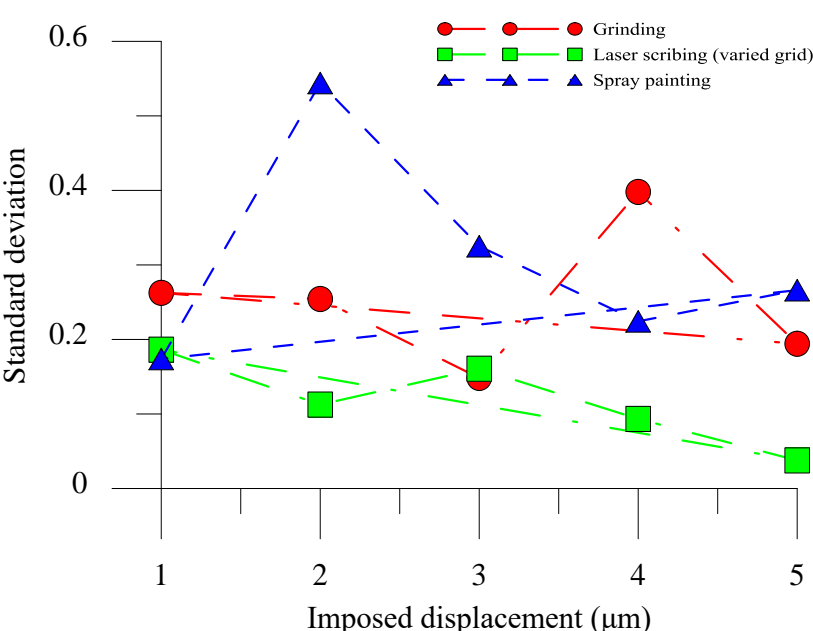

Fig. 6 Standard deviation of measured horizontal displacement for the five speckle patterns using $61 \times 61$ pixels subset (along the horizontal line of the image).

\subsection{Influence of the thermal strain of laser scribing}

To investigate the level of the strain induced by the laser scribing, a ring-core-based groove in the surface of the workpiece is cut to release the residual strain inside the core. The ring-core method is a partially destructive test 
method sensitive for measuring residual strain [13,14]. The entire experimental system was placed on the stage of a Sodick AP1L Micro Precision Electrical Discharge Machining machine (EDM) (Sodick, Japan). To examine the residual strain induced within a workpiece by laser scribing, the workpiece was annealed before performing the EDM hollow hole drilling and the surface of the workpiece was patterned by laser scribing. The thermal strain was measured by correlating the images captured at each temperature with the reference image at $25^{\circ} \mathrm{C}$ using a DIC software program (VIC-2D 2017 Digital Image Correlation, ver. 6.0.6; Correlated Solutions Inc., Columbia, SC). The experimental results in Fig. 7 show the additional strain induced by laser scribing under working parameters of $105 \mathrm{~V} / 0.007$ $\mu \mathrm{F} / 3 \mu \mathrm{s} / 6 \mu \mathrm{s}$ (open circuit voltage/discharge capacitance/pulse-on duration/pulse-off duration). It is observed that the peak strain is located in the outer ring and the strain decreases inward toward the inner core. During the EDM process, the machined outer ring surface is exposed to plasma with a high temperature; the EDM process can cause major deformation at the outer ring sections. Meanwhile, the deformation decreased in a direction radially inward of the ring. The thermal effect produced by laser scribing a speckle pattern was characterized as $25 \mu \varepsilon$ (Von Mises Strain). The magnitude of the induced strain is in the order of $5 \mathrm{MPa}$ with Young's modulus $200 \mathrm{GPa}$, which is within the uncertainty of residual stress measurement [15].

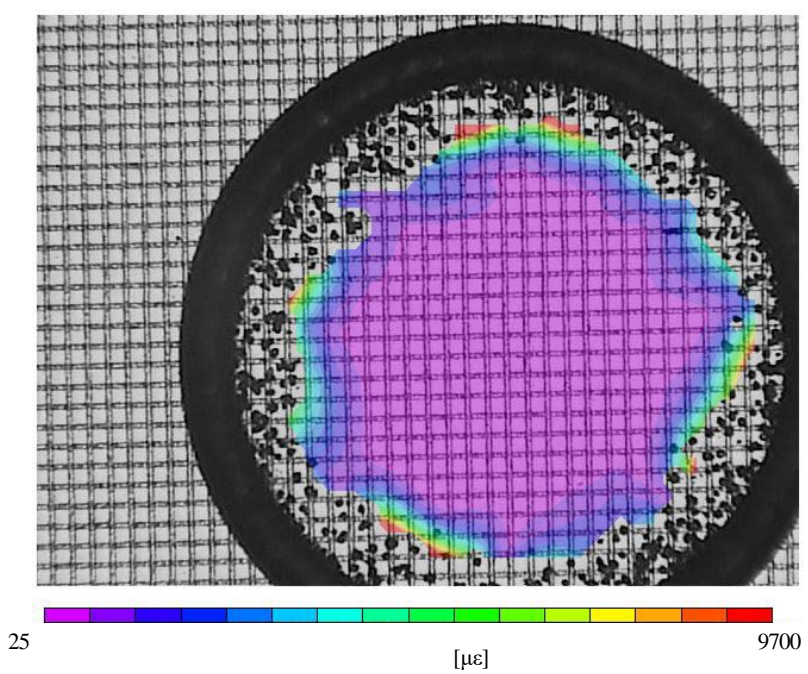

Fig. 7 Additional thermal strain analysis of laser scribing.

\section{Conclusions}

Laser scribing patterning is fast, inexpensive, robust, and appealing for digital image correlation measurement. Three speckle patterns were fabricated in this work, and verified with displacement accuracy in the actual translation experiments. It is shown that the speckle pattern produced with laser scribing has highest mean intensity gradient and high entropy values, and hence produces the lowest standard deviation error. The speckle pattern produced with laser scribing is proven to be of the best quality of the entire speckle patterns used in DIC in this work. The thermal effect of the patterns produced by laser scribing was characterized by measurement of the thermal strain under EDM ring-core machining.
AcknowledgmentsThe work was supported by the Ministry of Science and Technology, Taiwan, R.O.C. MOST 105-2622-E-027-020-CC3 and 105-2221-E-027-137.

\section{References}

[1] T. Chu, W. Ranson, and M. A. Sutton, Exp. Mech., 25, (1985) 232.

[2] G. Crammond, S. Boyd, and J. Dulieu-Barton, Opt Laser Eng., 51, (2013) 1368.

[3] J. Quinta da Fonseca, P. Mummery, and P. Withers, J. Microsc., 218, (2005) 9.

[4] D. Lecompte, A. Smits, S. Bossuyt, H. Sol, J. Vantomme, D. Van Hemelrijck, and A. Habraken, Opt. Laser Eng., 44, (2006) 1132.

[5] C. Sebastian, and E. Patterson, Exp. Mech., 39, (2015) 21.

[6] G. Lionello, and L. Cristofolini, Meas. Sci. Technol., 25, (2014) 107001.

[7] A. Stournaras, K. Salonitis, P. Stavropoulos, G. Chryssolouris, Theoretical and experimental investigation of pulsed laser grooving process, Int. J. Adv. Manuf. Technol., 44, (2009) 114.

[8] P. L. Reu, W. Sweatt, T. Miller, and D. Fleming, Exp. Mech., 55, (2015) 9.

[9] S. Yaofeng, and J. H. Pang, Opt. Laser Eng., 45, (2007) 967.

[10] H. Haddadi, and S. Belhabib, Opt. Laser Eng., 46, (2008) 185.

[11] D. Zuev, O. Novodvorsky, A. Lotin, A. Shorokhova, O. Khramova, G. Untila, A. Poroykov, T. Kost, and A. Chebotareva, Proc. Int. Conf. on Advanced Laser Technologies, Gwatt, 1, (2012).

[12] B. Pan, Z. Lu, and H. Xie, Opt. Laser Eng., 48, (2010) 469.

[13] E. Valentini, A. Benincasa, L. Bertelli, Proc. 40th Int. Conf. on National Convention of the AIAS-Italian Stress Analysis Associations, Palermo, (2011) p.919.

[14] C.-C. Ho, Y.-J. Chang, J.-C. Hsu, C.-L. Kuo, S.-K. Kuo, G.-H. Lee, Inventions, 1, (2016) 1010004.

[15] X. Huang, Z. Liu, H. Xie, Recent progress in residual stress measurement techniques, Acta. Mech. Solida. Sin., 26, (2013) 570.

(Received: May 8, 2017, Accepted: August 1, 2017) 